\title{
The role of emotional interference on learning in an emotional probabilistic Go/No-Go task
}

\author{
Rahmi Saylik $^{\circledR}$, Santiago Castiello ${ }^{2}{ }^{\circledR}$, Robin A Murphy $^{2} \odot$ \\ 'Mus Alparslan University, Department of Psychology, Mus - Turkey \\ ${ }^{2}$ University of Oxford, Department of Experimental Psychology, Oxford - UK
}

\begin{abstract}
Objective: Reversing learned associations interferes with previously acquired learning, a form of retroactive interference for the previous association and proactive interference on the new learning. We examined associations involving emotional content and how they might impact interference. The current study aims to discover the role positive, negative, and nonemotional stimuli play during acquisition and reversal learning in a probabilistic go/no-go task.

Method: The task consisted of separate conditions of happy, sad, angry, fearful emotional stimuli and non-emotional stimuli during separate acquisition and reversal training periods. Ninety-seven participants aged 18-35 (49 females) took part in the study.

Results: The results revealed that overall, participants were more accurate during acquisition than reversal. Further, happy stimuli were learned with greater accuracy during acquisition but were no easier to learn in reversal, effectively accompanied by a greater reversal cost.

Conclusion: There is evidence that happy emotional stimuli act like stimuli with a stronger learning rate much like learning of other high salience stimuli. Emotion valanced stimuli like other types of stimuli and can be described mechanistically by varying learning rate parameters of associative models.
\end{abstract}

Keywords: Acquisition, emotion, probabilistic go/no-go task, reversal

\section{INTRODUCTION}

People learn the associations between events that result in either positive or negative outcomes (1). These acquired associations guide our behaviors and form the basis of our causal understanding $(2,3)$. To maintain flexibility, changes in our environment require us to update or reverse learning by re-establishing new contingencies that sometimes oppose the original learning $(1,4,5)$. There is good evidence that association acquisition follows the principles of error correction. One prediction of error correction mechanisms is that if learning is faster and achieves asymptote more quickly, then it will also be more difficult to change or reverse that association (6). Associative models capture this effect on the basis of a free parameter for stimulus (7). This effect contrasts with interpretations of learning based on rule acquisition which might predict that people can switch associations more quickly if the content is acquired more strongly, perhaps if the stimulus dimensions are more distinguishable. A body of research has examined

How to cite this article: Saylik R, Obesso SC, Murphy RA. The role of emotional interference on learning in an emotional probabilistic Go/No-Go task. Dusunen Adam The Journal of Psychiatry and Neurological Sciences 2021;34:23-31.

Correspondence: Rahmi Saylik, Mus Alparslan University, Department of Psychology, Mus - Turkey 
these processes suggesting that reversing learned associations interferes with previously acquired learning a form of proactive interference $(4,5,8,9)$. We sought to vary the emotional salience of contingent outcomes to explore whether learning $t$ emotionally valent associations is captured by an associative process, and thereby showing stimulus salience differences, or is captured by a faster rule-based system. Researches involving the role of emotional stimuli in contingency learning remains widely unexplored although some research implies emotional stimuli, particularly stimuli expressed by happy facial expressions might involve a separate learning system (10). Reversal learning of emotional stimuli is of particular importance to help us understand how emotional memories are created and reduce unnecessary post-traumatic emotional pain, counterconditioning is one such example (11). This study aims to investigate the effects of emotions in the learning and reversal processes. Associative learning is defined as the process in which two events establish a predictive or informative relationship between them $(12,13)$. People learn about the valence of neutral events, biologically relevant events, as well as events with emotive content (14-16). Reversal learning can be defined as a change in a pre-established relationship between events and their empowering value with behavioral consequences $(6,17)$. Reversal learning contributes to flexibility in behavior, under both social and non-social circumstances $(2,17)$. Flexibility is seen as a primary cognitive function, one that may involve brain circuits including the amygdala, anterior cingulate cortex (ACC), orbitofrontal cortex (OFC), and ventral frontostriatal $(4,5,18)$. The amygdala and rostral ACC have been linked to the establishment of stimulus-outcome associations, whereas the OFC and ventral frontostriatal areas have been involved in behavioral adaptation and flexibility based on contingency changes $(4,5,17)$.

Emotional stimuli may capture and hold attention more effectively because they are more salient than neutral stimuli and therefore attention is prioritized priority $(10,15,16,19)$. The attentional priority towards emotional stimuli allows faster encoding and retrieval of emotional stimuli (images, words) than nonemotional stimuli (e.g., objects, shapes; 19,20). This effect of emotional stimuli seems to be more evident for positive stimuli, i.e., happy facial images (21-23). Happy facial emotional stimuli are faster to obtain and responding is often more accurate compared with other facial emotional stimuli in emotion recognition tasks as well as facial detection tasks (10). Although it is worth cautioning that any direct comparison of the effectiveness of stimuli assumes that they are matched on emotional content levels (i.e., happiness, sadness) since any difference in responsivity may reflect the nature of the experimental stimuli rather than the nature of the emotion. Nevertheless, the question of interest is whether positive or emotionally appetitizing stimuli affect the process of establishing associations (acquisition) and re-establishing the associations (reversal learning) in the same way. Previous research on action and outcome generally supports the idea that happy emotional stimuli facilitate establishing associations compared to negative emotional or neutral stimuli $(8,9,22,23)$. However, the findings regarding the effect of emotional stimuli in the reversal process are inconsistent. Some studies implies that happy emotional stimuli facilitate learning in acquisition phases leading to a decrease in reversal performance in typical go/no-go tasks (23) as well as in reward learning tasks (21) compared to negative facial emotional stimuli. This research indicates that happy emotional stimuli are intrinsically salient (2123). Whereas, angry facial stimuli may be aversive, confirming the idea that happy emotional stimuli are easier to learn and relatively more difficult to disengage from during reversal learning (21-23). In contrast, other studies to examine reversal learning, report that happy emotional stimuli result in superior task performance as well as reversal during acquisation $(8,9)$. These studies found that when participants need to reverse their responses to happy facial stimuli, they are more accurate than negative facial stimuli $(8,9)$.. This finding contradicts standard associative models of how learning is reversed unless the reversal is assumed to be a new learned relationship rather than an inversion of an existing relationship. This later research concluded that, due to the aversive nature of threatening stimuli, it may be more difficult to modify establishing associations with threatening faces than relationships with non-threatening emotional stimuli. The disagreement in the literature may reflect the different experimental paradigms and different participant populations. These studies used either a typical go/no-go task (23), or reward-based associative learning tasks (21). Furthermore, some studies included participants from different age groups (8), potentially having different levels of real-world experience with emotive contingencies. These studies are informative about the influence of emotions regarding blocking or cue-reward learning processes 
for certain age groups. However, they may be insufficient to come to a conclusion about the influence of emotions on non-reward-related associative learning in neurotypical adults.

In this study, we examined the role of positive and negative emotional and non-emotional stimuli during acquisition and reversal learning in the probabilistic go/ no-go task in neurotypical adults. The hypothesis tested was whether happy emotional stimuli facilitate learning when compared to other stimuli, but during reversal participants will be slower to learn the reversal contingency, a finding consistent with associative models of emotional association.

\section{METHOD}

\section{Participants}

A hundred and six healthy university students volunteered to participate the study. Participants read the online version of the participant information sheet and consent form and agreed to participate in the study by ticking the consent box. Because the scope of the study covered neurotypical participants, we invited students randomly via emails, and then participants with a history of psychiatric or neurological disorders were excluded. Thus, based on the self-report surveys, 9 participants were excluded due to depression ( 5 females, 3 males) or epilepsy ( 1 male). The remaining participants had no current or previous psychiatric or neurological disorders, a requirement to take part in the study. The final sample contained ninety-seven participants aged 18-35 (49 females: Mean=21.3, standard deviation $[\mathrm{SD}]=3.76 ; 57$ males: Mean $=23.09$, $\mathrm{SD}=4.80$ ). The trial lasted 45 minute and participants received 10 Turkish liras for their participation. The study was reviewed and approved by the Central University Ethics Committee at the University of Oxford and permission was granted from the Faculty of Art and Sciences at Mus Alpaslan University for recruiting participants.

\section{Materials}

\section{Probabilistic Go/No-go Task}

The Probabilistic go/no-go tasks consisted of emotional and non-emotional conditions controlled by the Gorilla Experiment Builder (www.gorilla.sc, 24) running on a sixteen-inch screen laptop.

This experiment consisted of an adapted version of a probabilistic cued go/no-go reaction time (RT) task (25). The task consisted of five conditions: four emotional (happy, sad, angry, fearful) and one non-
Table 1: Specification of probability based on number of trials in the task

\begin{tabular}{lccc} 
Trial types & $\begin{array}{c}\text { Number of } \\
\text { trials }\end{array}$ & Target cue & $\begin{array}{c}\text { Number of } \\
\text { trials }\end{array}$ \\
\hline Go & 50 & I & 38 \\
no-go & 50 & II & 12 \\
& & I & 12 \\
\hline
\end{tabular}

Table 1 presents condition specification in the probabilistic go/no-go task

emotional condition. The presentation order of the conditions was counterbalanced.

Each condition consisted of two epochs: acquisition and reversal. In each epoch, the spacebar was designated for a 'go' press in relation to the target stimulus. Each epoch was identical regarding task structure, timing, parameters, trial orders as well as response demands, except for the stimuli used for the go/no-go cues. In one epoch, 50 trials were conducted and $50 \%$ of them were required to go press and the remaining $50 \%$ to stop the press. As the task design was based on probabilistic outcomes, $75 \%$ of the go stimuli (e.g., happy face) were associated with correct feedback (e.g., if the target was a happy face then, $75 \%$ of happy faces and $25 \%$ of neutral faces were linked to 'go' press and received a green tick), whereas $25 \%$ of target stimuli were linked to 'no-go' (e.g., if the target was a happy face, then $25 \%$ of happy faces and $75 \%$ of neutral faces were linked to no-go and a red cross outcome) (See Table 1).

Emotive go/no-go conditions consisted of faces. Facial emotional (happy, sad, angry, fearful) and neutral expressions of 10 individuals ( 5 female, 5 male) from the Radboud Faces Database (RaFD; [26]) were used as emotional and neutral face stimuli. These stimuli were matched for emotions and gender. During acquisition, each condition involved learning how a single emotive contingency was followed by 'correct' feedback on $75 \%$ (a green tick) of the trials and during reversal conditions, the contingency was switched to be true $\% 75$ of the neutral expression trials (See Fig. 1, Panel A). The conditions were presented at random. A set of control conditions were included with non-emotional go/no-go conditions consisting of symbols (e.g., triangles and squares). During acquisition the target stimulus was a triangle, whereas in the reversal epochs the contingency changed so that the target stimuli became a square (See Fig. 1, Panel B).

Stimuli were presented the center of the screen for $500 \mathrm{~ms}$ each. The inter-stimulus interval (ISI) was 1000 ms. A fixation cross was displayed in the center of the 


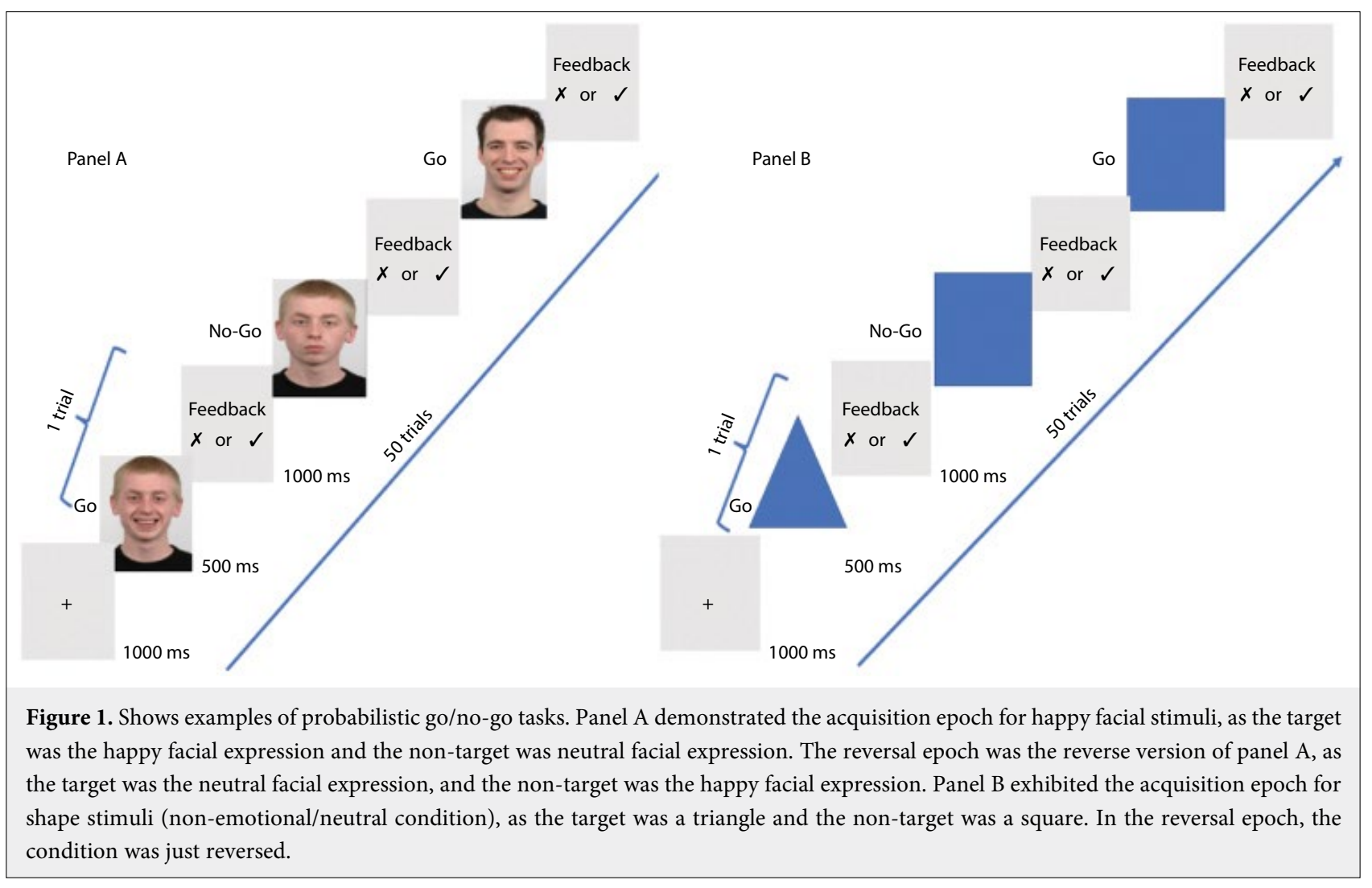

screen during the ISI. Instructions were displayed on the computer screen at the beginning of each block, and subjects were asked to press the enter key when they were ready to begin.

The response time for each trial and the number of correct responses across each condition was taken as the primary measures for that behavioral analysis.

\section{Procedure}

Before the experiment, all participants provided consent for participation and completed a self-assessment survey. The task was first introduced, and a short version was provided as to minimize sensory-motor or comprehension difficulties. All subjects fulfilled all conditions including acquisition and reversal epochs. In all epochs, participants were told to press the spacebar whenever they determined that a target go-stimulus was present based on their previous experience. Before each condition, the stimuli were introduced to the participants and they were informed about the target stimuli and the need to press go, while e the other stimulus was the 'no-go' stimulus. For the go-trials, participants had to to press the spacebar for the target stimuli and on 'no-go' trials, they were to withhold the action. Feedback was given for each trial. A green tick for the correct response and a red cross for $1000 \mathrm{~ms}$ appeared in the centre of the screen for the wrong response, although this feedback was proababilistic, and was only accurate on $75 \%$ of trials. Participants were instructed that they had to learn through trial-anderror, and as soon as they identified the target stimuli, they must hit the spacebar as quickly as possible, otherwise they would have to stop the non-target stimuli. The next trial started after the probabilistic feedback. Finally, the participants were informed upon the completion of the experiment.

\section{Statistical Analysis}

We performed three separate $5 \times 2$ factorial Analysis of Variance (ANOVA) using Jamovi 1.1.9.0 (27) with the first level within-subject factor stimuli manipulation (happy vs shapes vs. sad vs angry vs fear) and the second level within the subject factor group (acquisition vs reversal). Following this analysis, we also calculated $2 \mathrm{X} 2$ factorial ANOVA to compare the happy ones with each of the other stimuli separately. In other words, the Happy was compared with each of the shape and negative emotional stimuli separately. This is how we conducted the analysis to show how the learning process during acquisition and reversal is affected by happy stimuli compared to other stimuli. The dependent variables were response times and error rates. Post hoc 


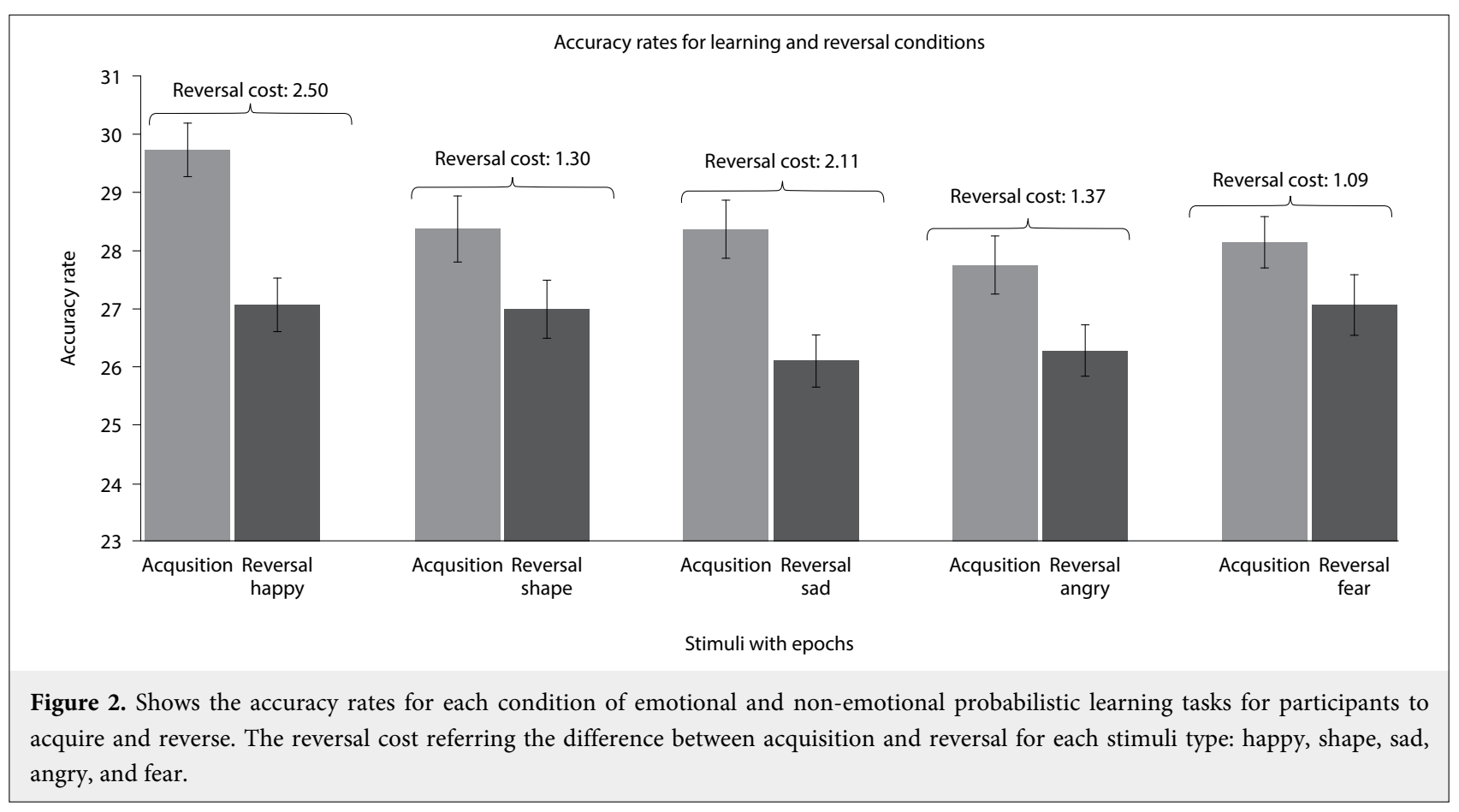

results reported using Tukey test and an alpha of .05 were used unless otherwise stated.

\section{RESULTS}

Figure 2 displays the accuracy rates for acquisition and reversal epochs across stimulus manipulation. In general, the figure suggests that acquiring the response (go) for happy faces was achieved with greater accuracy than for other stimuli. During reversal learning, all conditions were impaired relative to the acquisition, but there was little evidence that the contingencies of initiation or reversal were learned with fearful faces. 2X5 ANOVA (happy vs shape vs sad vs angry vs fear) X (acquisition vs reversal) was performed and no interaction effect was found ( $F[5,91]=1.66 ; p=0.157)$ although the main effects were significant for the F-type stimulant $(F[5,91]=3.18 ; \mathrm{p}=0.014)$ and reversal learning $(F[5,91]=26.65 ; \mathrm{p}<0.001)$.

\section{Happy vs Shape}

To understand the nature of the stimulus effects, we conducted a separate $2 \times 2$ ANOVA comparing Happy to each other stimulus type. The results demonstrated that the reversal cost did not reach the significance threshold for happy comparing the shape stimuli suggesting that both were acquired equally well, with no interaction ( $F[2,94]=3.52 ; \mathrm{p}=0.064)$. Further, while the main effects regarding reversal were significant suggesting that the accuracy was lower during reversal $(F[2,94]=27.34 ; p=0.001]$, there was no difference in accuracy for Happy faces and Shapes ( $F[2,94]=0.58 ; \mathrm{p}=0.446)$.

\section{Happy vs Sad}

The comparison of happy and sad faces demonstrated that a similar effect was a non-significant interaction effect $(\mathrm{F}[2,94]=1.74 ; \mathrm{p}=0.190)$ but strong evidence of lower performance during reversal for both faces ( $\mathrm{F}[2$, $94]=21.70 ; p=0.001)$ and remarkably lower overall accuracy for sad faces $(\mathrm{F}[2,94]=8.42 ; \mathrm{p}=0.005)$.

\section{Happy vs Angry}

The results for the happy comparing to angry stimuli found marginal significant interaction effects ( $F[2$, $94]=4.10 ; \mathrm{p}=0.046)$ but strong evidence for reduced accuracy during reversal $(F[2,94]=22.74 ; \mathrm{p}=0.001)$ and lower accuracy for angry faces compared to happy one $(\mathrm{F}[2,94]=8.96 ; \mathrm{p}=0.004)$.

\section{Happy vs Fearful}

Finally, the results for the happy comparing to fear suggested a lower level of accuracy for fearful faces during both acquisition and reversal. The overall interaction was significant $(\mathrm{F}[2,94]=5.41 ; \mathrm{p}=0.022)$. While the main effect was significant for reversal ( $\mathrm{F}[2$, $94]=22.74 ; \mathrm{p}=0.001$ ), the main effect of stimulus type was not significant $(F[2,94]=3.58 ; \mathrm{p}=0.060)$. The interaction was evaluated and described below as a series of post-hoc tests. 
Table 2: Mean and standard deviations across the epochs

\begin{tabular}{lcccc} 
Condition & Epochs & Mean & SD & t-test (acquisition vs reversal) \\
\hline Happy & Acquisition & 29.73 & 4.72 & $\mathrm{t}(96)=4.52, \mathrm{p}<0.001$ \\
& Reversal & 27.06 & 4.79 & \\
Shape & Acquisition & 28.37 & 5.74 & $\mathrm{t}(96)=2.25, \mathrm{p}=0.013$ \\
& Reversal & 26.99 & 5.03 & \\
Sad & Acquisition & 28.36 & 5.01 & $\mathrm{t}(96)=2.64, \mathrm{p}=0.005$ \\
& Reversal & 26.09 & 4.59 & \\
Angry & Acquisition & 27.74 & 5.08 & $\mathrm{t}(96)=2.40, \mathrm{p}=0.009$ \\
& Reversal & 26.27 & 4.56 & \\
Fear & Acquisition & 28.13 & 4.57 & $\mathrm{t}(96)=2.06, \mathrm{p}=0.024$ \\
& Reversal & 27.05 & 5.28 & \\
\hline
\end{tabular}

Table 2 presents the mean and standard deviation (SD) accuracy rates for epoch and paired t-tests for comparison of the acquisition and reversal epochs for each stimuli type

We performed a post-hoc tests for the acquisition and reversal epochs, comparing happy with the other stimuli separately, suggesting that participants in the acquisition epochs participants had significantly higher $\mathrm{r}$ accuracy in happy compared to sad ( $\mathrm{F}[2,94]=9.75$; $\mathrm{p}=0.002)$, happy compared to angry $(\mathrm{F}[2,94]=11.97$; $\mathrm{p}=0.001)$, happy compared to fear $(\mathrm{F}[2,94]=11.96$; $\mathrm{p}=0.001]$. Although a very similar result pattern was found for happy compared to shape stimuli, it did not reach significance threshold $(\mathrm{F}[2,94]=3.27 ; \mathrm{p}=0.074)$. On the other hand, none of these comparisons were significant for reversal epochs all $\mathrm{F}<1.23$, all $\mathrm{p}>0.270$ (i.e., an alpha of .01 was used).

The results of the main effect for reversal from the omnibus ANOVA suggested that participants were less accurate during the reversal for each stimulus. We performed a post-hoc test for each pair (e.g. happy acquisition vs happy reversal, shape acquisition vs shape reversal) shown separately as paired t-tests in Table 2 . This showed a significant drop in performance. The paired t-test results suggest lower and similar accuracy for all 5 stimulus types during reversal.

Taken together, the results suggest that the accuracy is different in different emotional expressions, but less learned during the reversal of all facial stimuli. The interaction effects representing the reversal cost (i.e., the differences between acquisition and reversal epochs) are significantly greater for the happy compared to shape, angry and fearful stimuli but not for the happy and sad stimuli. The significant main effects for reversal suggest that reversing the learning from acquisition to reversal epochs is a significant moderator for all comparisons with happy stimuli, whereas the main effects of stimuli were only a significant moderator for the happy compared to sad and angry, but not for fear and shape stimuli.
Although we found a similar result pattern with accuracy rates for the response times analysis, not all main effects and interaction effects were significant $\mathrm{F}<2.84$, all $\mathrm{p}>0.060$.

\section{DISCUSSION}

In this study, we investigated the role of emotional and neutral stimuli in the the emotive learning process in a go/no-go task. When examining acquisition and reversal timing, we found that participants applied the conditions with happy facial stimuli more accurately than angry or sad stimuli, but not the fearful ones during acquisition. For all groups, learning was more accurate during the acquisition than the reversal, suggesting some sort of interference. We demonstrated that participants were less accurate during reversal learning compared to acquisition, regardless of stimulus manipulation. Furthermore, in the reversal epochs the accuracy of happy facial stimuli decreased, suggesting that transforming every stimulus from a go cue to a no-go situation was difficult to even for a well-learned happy stimulus. Thus, all the reversal epochs showed similar accuracy rates. These results were accompanied by a greater reversal cost of conditions with happy facial stimuli compared to other stimuli except for the sad stimuli.

Distinctive effects of happy emotional stimuli have been repeatedly reported to be observed in associative learning tasks $(8,9,22,23)$. It has been highlighted that positive emotional stimuli, particularly happy facial expressions are more positive so that people are more likely to associate with happy facial stimuli $(8,9,21)$. The observation that participants acquired associations with happy stimuli (i.e., acquisition epochs) more accurately 
than negative emotional (i.e., sad and angry) as well as neutral (i.e., shapes) stimuli might therefore be taken as an evidence that happy emotional stimuli improved establishing associations compared to other stimuli. Previous associative learning studies often investigated the effects of happy stimuli in a specific negative stimulus, either angry or fearful, and suggested differing processes depending on the stimulus type. Our findings are consistent with an improved processing effect for happy stimuli compared to well-known negative emotional stimuli (i.e., sad, angry fearful) as well as neutral stimuli.

Regarding reversal or task switching learning, previous research suggests that happy emotional stimuli somehow affect the reversal process comparing certain negative emotional stimuli, but differing in the direction of the effect $(8,9,22,23)$. For instance, one study found that happy facial stimuli improve the establishment of associations when the target is (Go) and the fearful or neutral stimuli is the non-target (No-go), whereas when the situation was reversed and the happy facial stimuli became the non-target (No-go) and the other stimuli became the target (Go), participants had trouble blocking the non-target stimuli, as evident by a greater number of errors and response times (23). Although this study was conducted with a typical go/no-go task rather than a probabilistic go/no-go, it was informativeabout the effect of happy stimuli in reversal learning. In contrast, another study showed that happy emotional stimuli lead to better performance in acquisition as well as reversal learning compared to angry emotional stimuli (8). Our findings revealed that the accuracy rate in the reversal epochs with happy facial stimuli was not statistically different from negative emotional or nonemotional stimuli. Any superiority for happy emotional stimuli in the reversal process was not addressed in this study. In this regard, our findings for reversal were not consistent with the studies suggesting the superiority (8) or adverse impact (23) of happy emotional stimuli in the reversal epochs. One possible reason for such a result could be that we used three negative emotional faces (sad, angry, fearful). This meant that our stimulus group was overwhelmingly negative, and this may have reduced the ability to respond to happy faces. Indeed, further experimental work altering the positivity of the test sample of stimuli would be a useful test for population value sensitivity.

One important way to capture the effects of reversal involved examining the reversal costs for the no go responses from the second epoch by calculated by subtracting accuracy rates of the reversal from the accuracy rate of acquisition epochs for each stimulus type. We found that generally, reversal costs were higher for happy stimuli compared to other stimuli other than the sad facial stimuli. This indicates that the difference between the acquisition and reversal epochs with happy stimuli related to performance is larger than the difference in epochs of most of the other stimuli in this study. This may be a sign that disinhibition is associated with happy stimuli, as suggested earlier by some authors (23). However, as assessed in the above paragraph when the reversal epochs were examined separately, the performance was similar but not worse than the reversal epochs with neutral and negative stimuli. All this suggests that the higher costs associated with happy stimuli reflect stronger accuracy in the first phase rather than poor performance in the second phase. The mean accuracy in the second phase across all stimulus typeswas max $60 \%$-min $55 \%$ out of 50 trials, and this is consistent with above-chance performance, suggesting that participants learned to inhibit, but the inhibition was not under stimulus control.Taken together, our interpretation of the acquisition epochs is consistent with studies that found that happy emotional stimuli facilitate learning during acquisition $(8,9)$. Regarding the reversal, we observed that the performance in happy stimuli epochs become similar to the epochs with other stimuli. Due to the appetitive aspect of happy emotional stimuli, participants may be able to associate more quickly than negative emotional images. In the reversal epochs, this process may be negatively affected by the interference of acquisition training.

In conclusion, we found that participants were more accurate during acquisition epochs with happy facial stimuli than with sad or angry faces While the accuracy rate with happy stimuli has decreased significantly in times of reversal, it has never been inferior to those with other stimuli. On the other hand, thecost for reversal was higher for happy stimuli compared to other stimuli, suggesting that the difference in performance between the acquisition and reversal epochs was significantly greater than the difference for other stimuli. We interpret the findings as evidence that happy emotional stimuli facilitate establishing associations and could enhance learning in the acquisition process. This might be because happy emotional stimuli are inherently more pleasant as an appetitive stimulus so that it is more rapidly encoded and retrieved during the task process, which is reflected as better performance in establishing associations during acquisition. In contrast, there was no advantage 
for happy faces during reversal. It is difficult to say whether positive emotional stimuli inevitably facilitate learning in the acquisition, or it is simply taskdependent. Although the parameters such as task demand, difficulty, and response mappings were identical for each condition in this study the emotional stimuli in the probabilistic go / do task consisted of only real face images. Taken together, further studies should include emoticons, drawings, and other emotional images using various learning paradigms to validate whether the current findings could be generalized to all positive emotional stimuli in learning.

\begin{tabular}{|c|c|c|}
\hline \multicolumn{2}{|c|}{ Contribution Categories } & \multirow{2}{*}{$\begin{array}{l}\text { Author Initials } \\
\text { R.S., R.A.M. }\end{array}$} \\
\hline \multirow{3}{*}{ Category 1} & Concept/Design & \\
\hline & Data acquisition & R.S. \\
\hline & Data analysis/Interpretation & R.S., S.C.O. \\
\hline \multirow{2}{*}{ Category 2} & Drafting manuscript & R.S., S.C.O. \\
\hline & Critical revision of manuscript & S.C.O., R.A.M. \\
\hline Category 3 & Final approval and accountability & R.S., R.A.M. \\
\hline \multirow{2}{*}{ Other } & Technical or material support & R.A.M. \\
\hline & Supervision & R.A.M. \\
\hline
\end{tabular}

Acknowledgement: The second author (S.C.) thanks the University of Guadalajara for the research support.

Ethics Committee Approval: The study was reviewed and approved by the Central University Ethics Committee at the University of Oxford. (Number: AP099)

Informed Consent: Patient's were informed and written informed consent was obtained.

Peer-review: Externally peer-reviewed.

Conflict of Interest: No conflict of interest.

Financial Disclosure: Self funded.

\section{REFERENCES}

1. Byrom NC, Murphy RA. Individual differences are more than a gene $\times$ environment interaction: The role of learning. J Exp Psychol Anim Learn Cogn 2018; 44:36-55.

2. Aisbitt GM, Murphy RA. An Application of a Theory of Attention (Mackintosh, 1975) to Psychopathy: Variability in the Associability of Stimuli: In Trobalon JB, Chamizo VD, editors. Associative learning and cognition : homage to professor N. J. Mackintosh. In Memoriam (1935-2015). Barcelona: Edicions de la Universitat Barcelona 2016; 89-108. (Spanish)

3. Msetfi RM, Byrom N, Murphy RA. To neglect or integrate contingency information from outside the task frame, that is the question! Effects of depressed mood. Acta Psychol (Amst) 2017; 178:1-11.

4. Kringelbach ML, Rolls ET. Neural correlates of rapid reversal learning in a simple model of human social interaction. Neuroimage 2003; 20:1371-1383.
5. Cools R, Clark L, Owen AM, Robbins TW. Defining the neural mechanisms of probabilistic reversal learning using eventrelated functional magnetic resonance imaging. J Neurosci 2002; 22:4563-4567.

6. Brazil IA, Maes JH, Scheper I, Bulten BH, Kessels RP, Verkes RJ, et al. Reversal deficits in individuals with psychopathy in explicit but not implicit learning conditions. J Psychiatry Neurosci 2013; 38:E13-20.

7. Tortosa MI, Strizhko T, Capizzi M, Ruz M. Interpersonal effects of emotion in a multi-round Trust Game. Psicológica 2013; 34:179-198.

8. Nashiro K, Mather M, Gorlick MA, Nga L. Negative emotional outcomes impair older adults' reversal learning. Cogn Emot 2011; 25:1014-1028.

9. Willis ML, Palermo R, Burke D, Atkinson CM, McArthur G. Switching associations between facial identity and emotional expression: a behavioural and ERP study. Neuroimage 2010; 50:329-339.

10. Gutiérrez-Cobo MJ, Luque D, Most SB, Fernández-Berrocal P, Le Pelley ME. Reward and emotion influence attentional bias in rapid serial visual presentation. Q J Exp Psychol (Hove) 2019; 72:2155-2167.

11. Osborne-Crowley K, McDonald S, Rushby JA. role of reversal learning impairment in social disinhibition following severe traumatic brain injury. J Int Neuropsychol Soc 2016; 22:303313.

12. Baker AG, Murphy RA, Vallée-Tourangeau F. Associative and normative models of causal induction: Reacting to versus understanding cause. In Shanks DR, Holyoak, Medin DL, editors. The Psychology of Learning and Motivation San Diego: Academic Press, 1996; 1-45.

13. Ward RD, Gallistel CR, Balsam PD. It's the information! Behav Processes 2013; 95:3-7.

14. Murphy RA, Schmeer S, Vallée-Tourangeau F, Mondragón E, Hilton D. Making the illusory correlation effect appear and then disappear: the effects of increased learning. Q J Exp Psychol (Hove) 2011; 64:24-40.

15. Spiers HJ, Love BC, Le Pelley ME, Gibb CE, Murphy RA. Anterior temporal lobe tracks the formation of prejudice. J Cogn Neurosci 2017; 29:530-544.

16. Le Pelley ME, Reimers SJ, Calvini G, Spears R, Beesley T, Murphy RA. Stereotype formation: biased by association. J Exp Psychol Gen 2010; 139:138-161.

17. Schulz KP, Fan J, Magidina O, Marks DJ, Hahn B, Halperin JM. Does the emotional go/no-go task really measure behavioral inhibition? Convergence with measures on a non-emotional analog. Arch Clin Neuropsychol 2007; 22:151-160.

18. Budhani S, Marsh AA, Pine DS, Blair RJ. Neural correlates of response reversal: considering acquisition. Neuroimage 2007; 34:1754-1765.

19. Carretié L, Hinojosa JA, Martín-Loeches M, Mercado F, Tapia M. Automatic attention to emotional stimuli: neural correlates. Hum Brain Mapp 2004; 22:290-299. 
20. Sergerie K, Lepage M, Armony JL. A face to remember: emotional expression modulates prefrontal activity during memory formation. Neuroimage 2005; 24:580-585.

21. Averbeck BB, Duchaine B. Integration of social and utilitarian factors in decision making. Emotion 2009; 9:599-608.

22. Evans S, Shergill SS, Averbeck BB. Oxytocin decreases aversion to angry faces in an associative learning task. Neuropsychopharmacology 2010; 35:2502-2509.

23. Hare TA, Tottenham N, Davidson MC, Glover GH, Casey BJ. Contributions of amygdala and striatal activity in emotion regulation. Biol Psychiatry 2005; 57:624-632.
24. Anwyl-Irvine AL, Massonnié J, Flitton A, Kirkham N, Evershed JK. Gorilla in our midst: An online behavioral experiment builder. Behav Res Methods 2020; 52:388-407.

25. Fillmore MT, Rush CR. Polydrug abusers display impaired discrimination-reversal learning in a model of behavioural control. J Psychopharmacol 2006; 20:24-32.

26. Langner O, Dotsch R, Bijlstra G, Wigboldus DHJ, Hawk ST, van Knippenberg A. Presentation and validation of the radboud faces database. Cogn Emot 2010; 24:1377-1388.

27. Sahin MD, Aybek EC. Jamovi: An Easy to Use Statistical Software for the Social Scientists. Int J Assess Tools Educ 2019; 6:670-692. 